\title{
The demagnetizing field of a non-uniform rectangular prism
}

Smith, Anders; Nielsen, Kaspar Kirstein; Christensen, Dennis; Bahl, Christian Robert Haffenden; Bjørk, Rasmus; Hattel, Jesper Henri

Published in:

Journal of Applied Physics

Link to article, DOI:

$10.1063 / 1.3385387$

Publication date:

2010

Document Version

Publisher's PDF, also known as Version of record

Link back to DTU Orbit

Citation (APA):

Smith, A., Nielsen, K. K., Christensen, D., Bahl, C. R. H., Bjørk, R., \& Hattel, J. H. (2010). The demagnetizing field of a non-uniform rectangular prism. Journal of Applied Physics, 107(10), 103910.

https://doi.org/10.1063/1.3385387

\section{General rights}

Copyright and moral rights for the publications made accessible in the public portal are retained by the authors and/or other copyright owners and it is a condition of accessing publications that users recognise and abide by the legal requirements associated with these rights.

- Users may download and print one copy of any publication from the public portal for the purpose of private study or research.

- You may not further distribute the material or use it for any profit-making activity or commercial gain

- You may freely distribute the URL identifying the publication in the public portal

If you believe that this document breaches copyright please contact us providing details, and we will remove access to the work immediately and investigate your claim. 


\title{
The demagnetizing field of a nonuniform rectangular prism
}

\author{
A. Smith, ${ }^{1}$ K. K. Nielsen, ${ }^{1,2, a)}$ D. V. Christensen, ${ }^{1,3}$ C. R. H. Bahl, ${ }^{1}$ R. Bjørk, ${ }^{1}$ and \\ J. Hattel $^{2}$ \\ ${ }^{1}$ Fuel Cells and Solid State Chemistry Division, Ris $\phi$ National Laboratory for Sustainable Energy, \\ Technical University of Denmark, Frederiksborgvej 399, DK-4000 Roskilde, Denmark \\ ${ }^{2}$ Department of Mechanical Engineering, Technical University of Denmark, Building 425, \\ Niels Koppels Alle, DK-2800 Kongens Lyngby, Denmark \\ ${ }^{3}$ The Niels Bohr Institute, University of Copenhagen, Blegdamsvej 17, DK-2100 Copenhagen, Denmark
}

(Received 8 February 2010; accepted 12 March 2010; published online 20 May 2010)

The effect of demagnetization on the magnetic properties of a rectangular ferromagnetic prism under nonuniform conditions is investigated. A numerical model for solving the spatially varying internal magnetic field is developed, validated, and applied to relevant cases. The demagnetizing field is solved by an analytical calculation and the coupling between applied field, the demagnetization tensor field, and spatially varying temperature is solved through iteration. We show that the demagnetizing field is of great importance in many cases and that it is necessary to take into account the nonuniformity of the internal field, especially for nonconstant temperature distributions and composite magnetic materials. (C) 2010 American Institute of Physics. [doi:10.1063/1.3385387]

\section{INTRODUCTION}

The importance of demagnetization for the properties of a magnetic body has long been recognized. The long-range nature of the dipolar force acting between individual magnetic moments will give rise to a demagnetizing field inside the body and can give rise to shape dependence of the thermodynamic properties, e.g., the heat capacity, of the body. ${ }^{1}$ Only in uniform ellipsoidal samples (and a few other limiting cases such as an infinite sheet or an infinite cylinder) is the demagnetizing field uniform. Even in these cases, calculations of the demagnetizing field can be quite involved. ${ }^{2}$ The results can be expressed in terms of a demagnetization tensor $\mathbb{N}$

$$
\mathbf{H}=\mathbf{H}_{\mathrm{appl}}-\mathbb{N} \cdot \mathbf{M},
$$

where $\mathbf{H}$ is the total internal magnetic field, $\mathbf{H}_{\text {appl }}$ is the applied magnetic field, and $\mathbf{M}$ is the constant magnetization. The demagnetization tensor is symmetrical and has a trace equal to one.

If the coordinate axes are chosen to coincide with the principal axes of the ellipsoid, the demagnetization tensor becomes diagonal. Thus, the demagnetizing field is determined by three quantities $N_{x x}, N_{y y}$, and $N_{z z}$ whose sum is unity. When both the applied field and $\mathbf{M}$ are along a principal axis, Eq. (1) becomes the scalar equation

$$
H=H_{\text {appl }}-N M,
$$

where $N$ is the relevant demagnetization factor. This equation is often used for other geometries as well. In such cases $N$ should be interpreted as an average demagnetization factor. ${ }^{3}$ This approach can be sufficient if one is only interested in the average demagnetizing field over the entire sample. Otherwise, it becomes necessary to consider the spatial variation in the demagnetization explicitly. In particular, this is the

${ }^{a)}$ Electronic mail: kaki@risoe.dtu.dk. case when the relevant physical properties of the material depend nonlinearly on the local field.

In cases where the demagnetizing field is nonuniform, the calculation of it is nontrivial. Since the magnetization of the sample at a given point is dependent on the local field, which in its turn depends on the entire magnetization of the sample, the demagnetizing field has to be calculated selfconsistently, e.g., through an iterative approach. Often, the simplifying assumption that the magnetization can be considered as constant and independent of the external field is made. In this case, calculations for a wide range of nonellipsoidal bodies have been carried out. ${ }^{4-7}$ In Ref. 4 the case of letting the direction (but not the magnitude) of the magnetization vary is considered and analytical expressions for the demagnetizing field to second order are given for a few special geometries.

To go beyond such simple magnetic equations of state requires numerical methods. For thin disks with cylindrical symmetry Ref. 8 calculated the demagnetizing field for homogeneous applied fields and four different magnetic equations of state: constant susceptibility, constant susceptibility with step discontinuity, hyperbolic tangential field-dependent susceptibility and finally the equation of state for a mean field ferromagnet. In Ref. 9 an axisymmetric model was applied to the problem of demagnetization in an active magnetic regeneration (AMR) device.

In this work we present a full three-dimensional modeling of a rectangular prism based only on the assumption of discretizing the prism into a mesh of grid cells each assumed to have a constant temperature and magnetization. A similar approach was followed in Ref. 10 however, only the demagnetization tensor was calculated and not the demagnetizing field.

The model is introduced in Sec. II. Then, in Sec. III the model is applied to the case of a flat prism with the magnetic field aligned along different axes and with different internal temperature distributions. Two main cases are considered: a 
single-material prism with an internal temperature gradient and a multimaterial prism, i.e., a single prism consisting of several materials, uniformly distributed for simplicity, each having an individual Curie temperature. In Sec. IV the model is compared to the average expression given in Ref. 3. The results, and in particular their relevance to the construction and optimization of an AMR magnetic refrigeration system where multiple materials are expected to be crucial for performance, are discussed in Sec. V.

\section{DEMAGNETIZATION MODEL OF A RECTANGULAR PRISM} form

The internal magnetic field can be written in the general

$$
\mathbf{H}=\mathbf{H}_{\mathrm{appl}}+\mathbf{H}_{\mathrm{dem}},
$$

where the difference between the internal and external field is the demagnetizing field $\mathbf{H}_{\mathrm{dem}}$.

The demagnetizing field can be expressed as an integral over the interior $\Omega$ of the body in the following manner

$$
\mathbf{H}_{\mathrm{dem}}(\mathbf{r}, T)=\frac{1}{4 \pi} \int_{\Omega} \mathrm{d} \mathbf{r}^{\prime} \mathrm{D}\left(\mathbf{r}-\mathbf{r}^{\prime}\right) \cdot \mathbf{M}\left[\mathbf{H}\left(\mathbf{r}^{\prime}, T\right), \mathbf{r}^{\prime}, T\right],
$$

where $\mathrm{D}$ is a symmetric $3 \times 3$ tensor whose components are given in Appendix A. This expression is valid both for points $\mathbf{r}$ inside and outside the body. The magnetization is in general a function of both the internal field, position and temperature. The explicit position dependence is relevant when, e.g., a multimaterial prism is considered. Due to the appearance of the internal field in $\mathbf{M}$, Eq. (4) becomes an implicit equation for the demagnetizing field. Only when the magnetization is independent of the internal field, the equation may be evaluated explicitly. For constant magnetization this may be done either by direct integration ${ }^{4}$ or through a Fourier transform approach. ${ }^{6,7}$

At low applied fields the magnetization within a soft ferromagnetic body will form domains in order to minimize the magnetostatic energy. Upon application of a modest magnetic field the domains will be aligned bringing the ferromagnet into a single-domain, saturated state. This saturated state is always assumed in the following.

To assume that the magnetization will not depend on the internal field will be a fair approximation for ferromagnetic bodies at temperatures far below the Curie temperature. However, close to the Curie temperature the magnetization has a strong field dependence. In the following, we will assume that the mean field equation of state captures the essential aspects of this dependence for the purpose of calculating the demagnetizing field. We do not expect our results to differ markedly for more realistic equations of state.

For concreteness we will only consider rectangular prisms. However, the procedure below may readily be adapted to, e.g., multimaterial spheres or cylinders. Considering now a rectangular prism bounded by the inequalities $-a \leq x \leq a,-b \leq y \leq b$, and $-c \leq z \leq c$ (see Fig. 1) the demagnetizing field may be expressed as

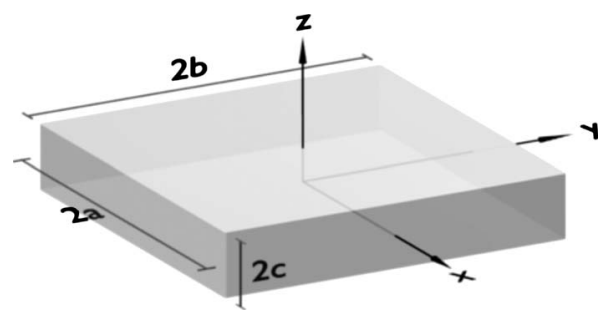

FIG. 1. The coordinate system of the modeled rectangular prism with the coordinate system defined with Origo at the center of the prism. Note that the $z$-direction is the "thin" direction, i.e., $2 c \ll 2 a$.

$$
\begin{aligned}
\mathbf{H}_{\mathrm{dem}}(\mathbf{r}, T)= & \frac{1}{4 \pi} \int_{-a}^{a} \mathrm{~d} x^{\prime} \int_{-b}^{b} \mathrm{~d} y^{\prime} \int_{-c}^{c} \mathrm{~d} z^{\prime} \mathrm{D}\left(\mathbf{r}-\mathbf{r}^{\prime}\right) \\
& \cdot \mathbf{M}\left\{\mathbf{H}\left[\mathbf{r}^{\prime}, T\left(\mathbf{r}^{\prime}\right)\right], \mathbf{r}^{\prime}, T\left(\mathbf{r}^{\prime}\right)\right\} .
\end{aligned}
$$

Dividing the prism into $n_{x} \times n_{y} \times n_{z}$ rectangular cells (following Refs. 10 and 11) the integral in Eq. (5) may be written as a sum of integrals over each cell

$$
\begin{aligned}
\mathbf{H}_{\mathrm{dem}}(\mathbf{r}, T)= & \frac{1}{4 \pi} \sum_{i=1}^{n_{x}} \sum_{j=1}^{n_{y}} \sum_{k=1}^{n_{z}} \int_{-a^{\prime}}^{a^{\prime}} \mathrm{d} x^{\prime} \int_{-b^{\prime}}^{b^{\prime}} \mathrm{d} y^{\prime} \int_{-c^{\prime}}^{c^{\prime}} \mathrm{d} z^{\prime} \\
& \mathbb{D}\left(\mathbf{r}-\mathbf{r}^{\prime}\right) \cdot \mathbf{M}\left\{\mathbf{H}\left[\mathbf{r}^{\prime}, T\left(\mathbf{r}^{\prime}\right)\right], \mathbf{r}^{\prime}, T\left(\mathbf{r}^{\prime}\right)\right\}
\end{aligned}
$$

with $a^{\prime}=a / n_{x}, b^{\prime}=b / n_{y}$, and $c^{\prime}=c / n_{z}$. Each cell has the same relative dimensions as the original prism.

Assuming each cell to be sufficiently small to have constant magnetization, $\mathbf{M}_{0}\left(\mathbf{r}_{i, j, k}^{\prime}, T_{i, j, k}\right)$, Eq. (6) may be approximated by

$$
\begin{aligned}
\mathbf{H}_{\mathrm{dem}}(\mathbf{r}, T) \approx & -\sum_{i=1}^{n_{x}} \sum_{j=1}^{n_{y}} \sum_{k=1}^{n_{z}} \\
& \mathbb{N}\left(\mathbf{r}-\mathbf{r}_{i, j, k}^{\prime}\right) \cdot \mathbf{M}_{0}\left[\mathbf{H}\left(\mathbf{r}_{i, j, k}^{\prime}, T_{i, j, k}\right), \mathbf{r}_{i, j, k}^{\prime}, T_{i, j, k}\right],
\end{aligned}
$$

where $\mathbb{N}$ denotes the symmetric $3 \times 3$ demagnetization tensor field with the components given in Eqs. (A8) and (A12) below. The vector $\mathbf{r}_{i, j, k}^{\prime}$ denotes the center of the cell with index $i, j, k$.

The magnetization is generally a function of both the magnitude of the internal field, $H$, and temperature, $T$. Therefore, Eq. (3) has to be solved by iteration. For simplicity the magnetization is assumed to be aligned with $\mathbf{H}$, i.e., there is no magnetocrystalline anisotropy. ${ }^{4}$

To obtain the magnitude of the magnetization, $M$, the mean field equation of state for a ferromagnet is assumed ${ }^{12}$

$$
M(T, H)=\mathrm{N}_{\mathrm{s}} g J \mu_{\mathrm{B}} \rho B_{J}(\chi)
$$

with $\mathrm{N}_{\mathrm{s}}$ denoting the number of magnetic spins per unit mass, $g$ the Landé factor, $J$ the total angular momentum in units of $\hbar, \mu_{\mathrm{B}}$ the Bohr magneton, and $\rho$ the mass density. The Brillouin function, $B_{J}$, is defined as

$$
B_{J}(\chi)=\frac{2 J+1}{2 J} \operatorname{coth}\left(\frac{2 J+1}{2 J} \chi\right)-\frac{1}{2 J} \operatorname{coth}\left(\frac{1}{2 J} \chi\right),
$$




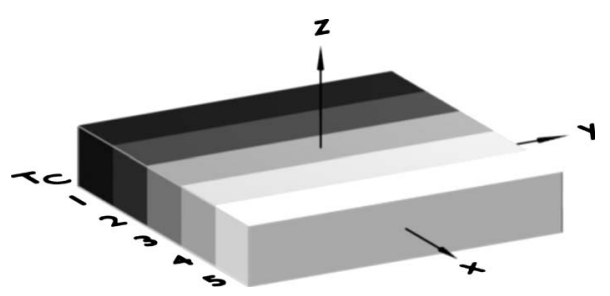

FIG. 2. The concept of grading the prism with different ferromagnets. In this case five materials are illustrated. The Curie temperatures differ from layer to layer as indicated by $T_{\mathrm{C} 1-5}$.

$$
\chi=\frac{g J \mu_{\mathrm{B}} \mu_{0} H}{k_{\mathrm{B}} T}+\frac{3 T_{\mathrm{C}} J}{T(J+1)} B_{J}(\chi) .
$$

Here the vacuum permeability, $\mu_{0}$, the Boltzmann constant, $k_{\mathrm{B}}$, and the Curie temperature, $T_{\mathrm{C}}$, were introduced. Equation (10) is iterated to obtain a self-consistent solution. In Appendix B a numerical model solving the coupled problem in Eqs. (3)-(10) is described in detail.

\section{APPLICATION OF THE MODEL}

Four different cases are investigated in the following. Two cases with a rectangular prism made of a single magnetic material, i.e., having one Curie temperature, and two cases with a so-called graded material, i.e., a composite material which contains regions with different Curie temperatures. In the latter case, the grading is assumed to be along the $x$-direction; for concreteness we consider five equal-sized regions each with its own Curie temperature (illustrated in Fig. 2). This is presented in Sec. III B.

Both materials configurations are considered under two different temperature situations: one with a constant temperature and one with an imposed temperature gradient. The latter case is relevant to investigate for, e.g., magnetic refrigeration, or in other cases where a thermal gradient is present in the system. In general, such a gradient may cause the prism to be in different magnetic phases at the same time. This is the typical operation mode of a magnetic material used in magnetic refrigeration, which will be roughly centered around the Curie temperature for optimal utilization of the magnetocaloric effect. ${ }^{13}$

As a magnetic material, gadolinium $(\mathrm{Gd})$ is used since it can be fairly well described by the mean field equation of state, Eq. (8) ${ }^{14}$ additionally it acts as a de facto benchmark material in magnetic refrigeration. The Curie temperature of Gd is taken to be $293 \mathrm{~K}$, and the other input parameters for the mean field equation of state are given in Table I. The dimensions of the prism are taken to be $2 a=0.02 \mathrm{~m}, 2 b$ $=0.02 \mathrm{~m}$, and $2 c=0.001 \mathrm{~m}$ in all cases. The coordinate system is illustrated in Fig. 1.

TABLE I. Parameters for the mean field equation of state, Eq. (8), for Gd. Data taken from Refs. 15 and 16.

\begin{tabular}{lccccc}
\hline \hline Parameter & $\begin{array}{c}\mathrm{N}_{\mathrm{s}} \\
\left(\mathrm{kg}^{-1}\right)\end{array}$ & $\begin{array}{c}g \\
(-)\end{array}$ & $\begin{array}{c}J \\
(\hbar)\end{array}$ & $\begin{array}{c}\rho \\
\left(\mathrm{kg} \mathrm{m}^{-3}\right)\end{array}$ & $\begin{array}{c}T_{\mathrm{C}} \\
(\mathrm{K})\end{array}$ \\
\hline Value & $3.83 \times 10^{24}$ & 2 & $7 / 2$ & 7900 & 293 \\
\hline \hline
\end{tabular}
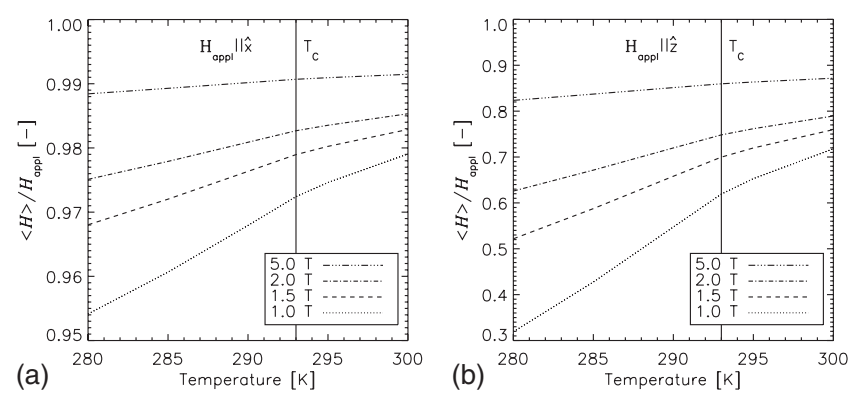

FIG. 3. The normalized mean of the magnetic field as a function of the (spatially constant) temperature for four different applied fields in the case of a rectangular prism consisting of one magnetic material. (a) The applied field is along the $x$-direction. (b) The applied field is along the $z$-direction.

\section{A. Single Curie-point flat prism \\ 1. Constant temperature}

We first consider the case of a single material with a constant temperature to validate our approach. This is a wellknown situation and will only briefly be discussed. In Fig. 3 the normalized mean of the magnitude of the internal field is plotted as a function of the (spatially constant) prism temperature for four different applied fields. It is evident from the figure that the effect of demagnetization decreases at higher applied fields in the ferromagnetic phase. This follows directly from the fact that the magnetization is saturated in the ferromagnetic phase and thus the demagnetizing field becomes constant. However, a field of more than $5 \mathrm{~T}$ is needed in order for this to be the case (this field value is material dependent, of course). Furthermore, when applying the field in the $x y$-plane of the prism, the magnetic field is reduced with a few percent whereas it is reduced with up to $70 \%$ in the case of applying the field along the $z$-direction. The decrease is dependent on temperature and material properties.

\section{Linear temperature profile}

In the following, the rectangular prism is assumed to have an imposed temperature profile ranging linearly from 280 to $300 \mathrm{~K}$ along the $x$-direction. This will make the magnetic state of the prism depend on $x$. This is a special case of great importance in, e.g., magnetic refrigeration where a magnetic material acts both as a regenerator material, i.e., upholding a thermal gradient, and as an active magnetic material through the magnetocaloric effect.

Figure 4 shows the two cases where the applied field is along the $x$-direction and $z$-direction, (a) and (b), respectively. Four different fields have been applied, namely 1.0, 1.5, 2.0, and 5.0 T. The same trends as in Fig. 3 are observed. The rather large applied magnetic field of $5 \mathrm{~T}$ saturates the magnetization (in the ferromagnetic phase) and the effect of demagnetization is thus small here. However, considering the cases of applied fields of 1.0-2.0 T a rather large gradient in the internal field is observed when the applied field is along the $z$-direction [Fig. 4(b)]. In the case of applying the field along the $x$-direction [Fig. 4(a)], the internal field is generally not affected greatly by the demagnetizing field. However, on the low temperature edge, i.e., where $x / a=-1$, the field drops rapidly. A similar, though not as large, drop is seen on the 

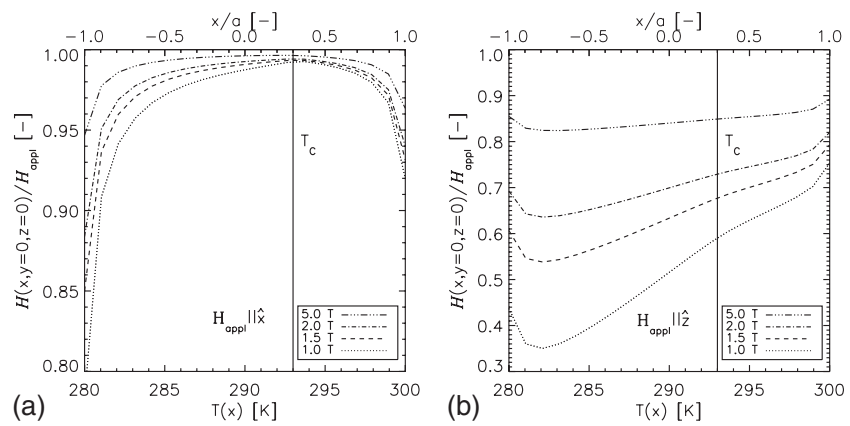

FIG. 4. The magnetic field along the line $y=0, z=0$, and $-1 \leq x / a \leq 1$. The prism has an imposed thermal gradient along the $x$-direction ranging from 280 to $300 \mathrm{~K}$ and consists of one magnetic material with a Curie temperature of $293 \mathrm{~K}$. (a) The applied field is along the $x$-direction. (b) The applied field is along the $z$-direction.

high temperature edge (at $x / a=1$ ). These two drops in the internal field are due to the fact that the demagnetization tensor field is largest on the edges perpendicular to the direction of the applied field. The reason that the lower temperature edge has the somewhat greater drop in internal field is because this part of the prism is in the ferromagnetic phase and thus the magnetization is largest here and consequently the demagnetizing field is greater.

\section{B. Flat prism with multiple Curie temperatures}

In the following a rectangular prism consisting of five equally distributed magnetic materials resembling $\mathrm{Gd}$ but with Curie temperatures 280 K, 285 K, 290 K, 295 K, and $300 \mathrm{~K}$, respectively, is considered. The grading of the prism is along the $x$-axis. With the temperature of the prism in the interval 280 to $300 \mathrm{~K}$ the individual parts of the prism will be in different magnetic phases but still in the vicinity of their respective Curie temperatures.

\section{Constant temperature}

Considering the case with the prism having a constant temperature the magnitude of the internal field across the prism in the direction of the grading is plotted in Fig. 5 for five different constant temperatures. The applied field is in all cases equal to $1 \mathrm{~T}$. The magnetic field is seen to be discontinuous in the $x$-direction when applying the field in this direction [Fig. 5(a)], whereas it is continuous in the $x$-direction when applying the field along the $y$-direction and $z$-direction [Figs. 5(b) and 5(c)]. This is to be expected since in the former case the magnetic field lines are crossing material boundaries and the normal component of $\mathbf{H}$ is discontinuous. In the latter cases the field lines are perpendicular to the materials boundaries and the parallel component of $\mathbf{H}$ across boundaries is continuous as expected. It should be noted that the largest component of $\mathbf{H}$ is along the direction of the applied field. Considering the magnetic flux density,

$$
\mathbf{B}=\mu_{0}(\mathbf{H}+\mathbf{M}),
$$

the opposite is true, i.e., the normal component is continuous whereas the parallel component is discontinuous. This is seen in Figs. 6(a) and 6(c). Figure 6(b) shows a plot of the magnitude of the magnetic flux density along the $x$-axis
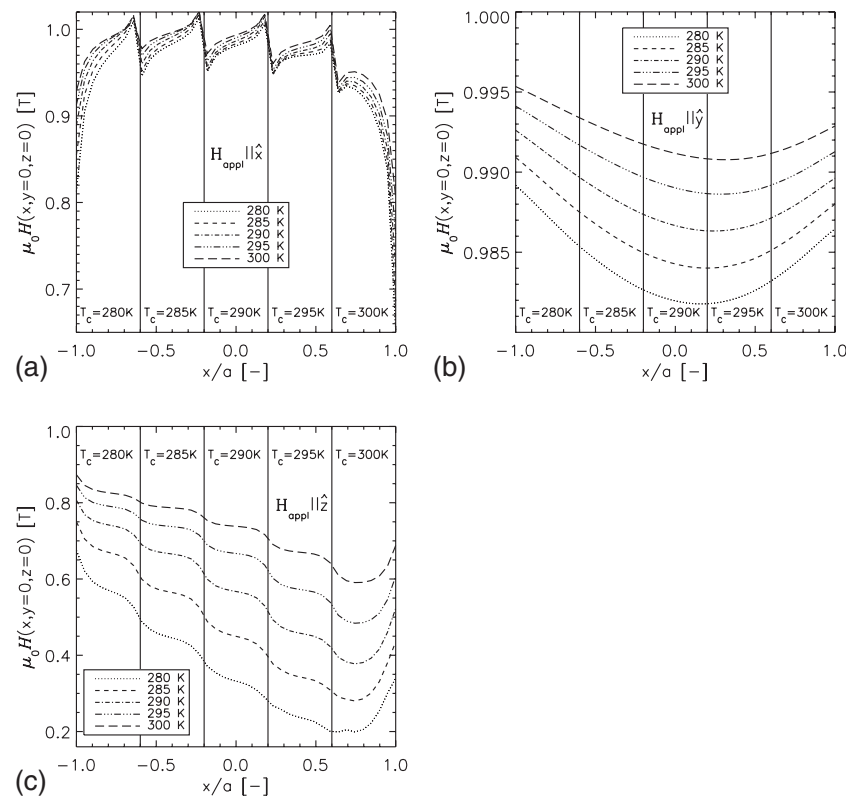

FIG. 5. The magnitude of the magnetic field along the line defined as -1 $\leq x / a \leq 1, y=0$, and $z=0$ through the prism for five different constant temperatures. The prism is divided in five regions each being a magnetic material resembling Gd but with different Curie temperatures $(280$ K, 285 K, 290 K, $295 \mathrm{~K}$, and $300 \mathrm{~K}$, respectively), as illustrated in Fig. 2. (a) The applied field is along the $x$-direction. (b) The applied field is along the $y$-direction. (c) The applied field is along the $z$-direction. In all cases $\mu_{0} H_{\text {appl }}=1.0 \mathrm{~T}$.

when the magnetic field is applied along the $y$-axis. Since $H$ is virtually constant (to within a few per mille; see Fig. 5), the magnetization is dominating the spatial variation in $B$. This is seen in the staircaselike plot on Fig. 6(b). At, e.g., a constant temperature of $280 \mathrm{~K}$, the value of $B$ in the part of the prism with a Curie temperature of $280 \mathrm{~K}$ is equal to $B$ at a temperature of $285 \mathrm{~K}$ in the part of the prism with a Curie
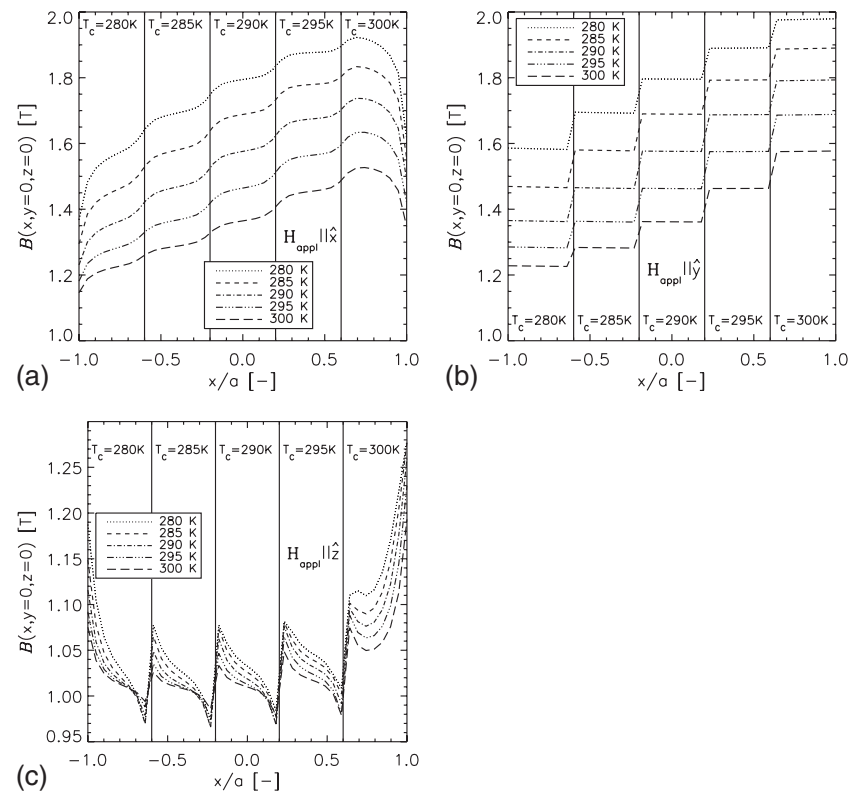

FIG. 6. The magnitude of the magnetic flux density, $B$, along the same line as in Fig. 5, i.e., $-1 \leq x / a \leq 1, y=0, z=0$ for five different temperatures. The prism is the same as considered in Fig. 5. (a) The applied field is along the $x$-direction. (b) The applied field is along the $y$-direction. (c) The applied field is along the $z$-direction. In all cases $\mu_{0} H_{\text {appl }}=1.0 \mathrm{~T}$. 
point of $285 \mathrm{~K}$, etc. The discontinuities across the internal materials boundaries are expected again due to the boundary conditions.

When applying the magnetic field along the $x$-direction, which causes minimal demagnetization, it is observed that the variation in the temperature of the prism does not change the internal field significantly. However, when applying the field along the $z$-direction, Fig. 5(c), a significant difference is observed between the various temperature cases. The lower the temperature of the prism the more of the individual composites are in their ferromagnetic state. This produces higher magnetization values and thus also a larger demagnetizing field. For increasing Curie temperature (along the $x$-axis) the magnetic field decreases because of the larger magnetization. It should be noted that for a constant temperature of $280 \mathrm{~K}$ the average internal field is about $60 \%$ of the applied field. The maximum decrease is observed to be around $80 \%$ for the cases studied here. The reason for the increase in magnetic field at either ends for all temperature cases is the relatively low demagnetization factor on the boundary. It is noted that the internal field may actually be greater than the applied field locally. This is seen in Fig. 5(a) and can be explained by flux shimming due to the discontinuity in the permeability on the boundary between two different magnetic materials. ${ }^{9}$

Finally, it is noted that applying the field along the $y$-direction [Fig. 5(b)] yields both a continuous and large internal magnetic field. The difference in this situation between the largest and smallest values of the magnitude of the internal field is only a few percent whereas in the case of applying the field along the $x$-direction may give a decrease in as much as $30 \%$, though only in relatively small regions.

\section{Linear temperature profile}

Figure 7 shows the magnetic field in the $x$-direction of a prism similar to the one considered in Sec. III B 1 but with an imposed linear temperature profile ranging from 280 to $300 \mathrm{~K}$. For the four different applied fields, 1, 1.5, 2.0, and 5.0 T, Figs. 7(a)-7(c) show the case when magnetizing along the $x$-direction, $y$-direction, and $z$-direction, respectively. A magnetic field similar to that obtained in the constant temperature case, Fig. 5, is produced in this case. However, when applying the field along the $x$-direction the drop in magnetic field at either end is similar to the edge defined as $x=-a$ in Fig. 5(a).

Again, as discussed in Sec. III B 1, applying the field along the $y$-direction [Fig. 7(b)] yields both a smooth and large internal field. This may be explained by the simple fact that the normal component of $\mathbf{H}$ is continuous across materials boundaries and the demagnetization is low when the field is applied in the $y$-direction.

Finally, when applying the field along the $z$-direction the internal field is more smooth than in the constant temperature case [see Figs. 5(c) and 7(c)]. This is due to the fact that each section of the prism having a specific Curie temperature is relatively close to this temperature. Thus, the magnetization across the prism is fairly constant as opposed to the decrease with increasing $x$ in Fig. 5(b). This results in a more constant demagnetizing field. It is also observed in Figs. 7(a)-7(c)
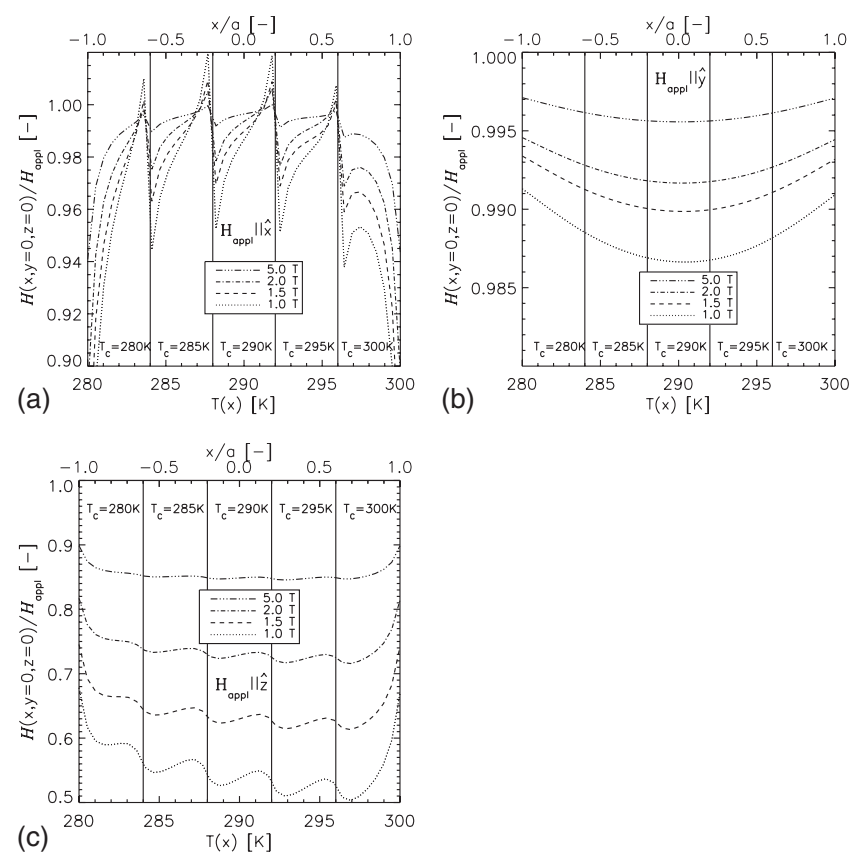

FIG. 7. The magnitude of the internal field along the line $-1 \leq x \leq 1, y=0$, and $z=0$ for four different applied fields. The prism is divided into five materials each having a different Curie temperature as in Fig. 5. The prism has an imposed linear temperature profile along the $x$-direction ranging from $280 \mathrm{~K}$ to $300 \mathrm{~K}$. (a) The applied field is along the $x$-direction. (b) The applied field is along the $y$-direction. (c) The applied field is along the $z$-direction.

that lower applied fields induce larger variation along the $x$-direction, which is due to the fact that the magnetization becomes saturated above a certain field and thus the demagnetizing field becomes constant.

\section{COMPARISON TO THE AVERAGE DEMAGNETIZATION FACTOR}

In Ref. 3 the average demagnetization factor, $N$, of a prism under the assumption that the magnetization and internal field are homogeneous and constant was calculated by Aharoni. In the following a comparison between the results of the model presented here and this average value is performed. Experimentally, the applied field and the mean magnetization along the direction of the applied field may be obtained. This leads to the definition of a representative average demagnetization factor, $N_{0, i}$

$$
\left\langle H_{i}\right\rangle=H_{\text {appl }, i}-N_{0, i}\left\langle M_{i}\right\rangle
$$

with the subscript $i$ denoting the component of the field. It should be noted that with this definition, $N_{0, i}$, is not the average of the demagnetization tensor field given in Eqs. (A8) and (A12). In particular, the sum of $N_{0, x}, N_{0, y}$, and $N_{0, z}$ need not be unity. It should rather be interpreted as a simplification of the model results in terms of a single number, which is useful when analyzing experimental data.

Figure 8(a) shows $N_{0, x}$ for the case of a constant temperature, single material rectangular prism with an applied field of $1 \mathrm{~T}$ along the $x$-direction. The prism has a symmetric $y z$-cross section and the length is varied in the $x$-direction giving rise to a variation in the aspect ratio. The average demagnetization factor is seen to coincide with the Aharoni 

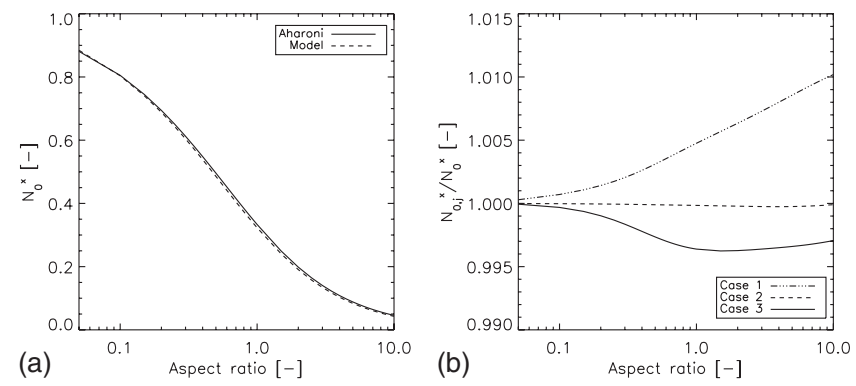

FIG. 8. The representative average demagnetization factor as defined in Eq. (12). (a) Shows this factor as a function of aspect ratio for a rectangular prism with quadratic cross section (in the $y z$-plane) and varying length (along the $x$-direction). The applied field is along the $x$-direction and has a magnitude of $1 \mathrm{~T}$. The temperature is fixed at $293 \mathrm{~K}$, i.e., the Curie temperature. (b) Three specific cases where the temperature and composition of the magnetic material are varied. Case 1 is for a constant temperature of 293 K with five materials, with Curie temperatures 280 K, 285 K, 290 K, 295 K, and $300 \mathrm{~K}$, respectively, spaced evenly along the $x$-direction. Case 2 is for the same composition as in Case 1 but with a linear temperature profile ranging from $280-300 \mathrm{~K}$. Case 3 is for a single material prism with an imposed linear temperature profile also from 280-300 K. In all cases the graphs show the ratio between the respective average demagnetization factor and the single material, constant temperature average demagnetization factor.

expression almost completely. In the limits where the aspect ratio goes to zero and infinity, respectively, the demagnetization factors are equal. However, for aspect ratios from one to five the Aharoni demagnetization factor is a few percent larger than the representative average defined in Eq. (12). This may be explained from the fact that the corners of the prism have a relative large impact on the demagnetization factor in these cases, i.e., the magnetization and thus internal field deviate mostly from being parallel to the applied field for this range of aspect ratios. Since the Aharoni expression assumes the magnetization to be completely parallel to the applied field, a discrepancy is to be expected.

Figure $8(\mathrm{~b})$ shows the average demagnetization factor for the constant temperature and single material case compared to the three cases: (1) a rectangular prism graded with five materials as discussed in Sec. III B 1, (2) same as in (1) but with an imposed linear temperature profile, i.e., as discussed in Sec. III B 2, and finally (3) a single material prism with an imposed linear temperature profile as discussed in Sec. III A 2. It is clearly evident from the figure that the representative average demagnetization factor is not purely geometric. In the cases investigated here the effect of grading the material or imposing a linear temperature profile is of the order of $1 \%$ only. This should be taken as a consequence of the selected cases rather than as a general rule. Indeed, Ref. 8 found their effective demagnetization factor to vary with as much as $10 \%-20 \%$ due to nongeometric factors.

\section{CONCLUSIONS AND DISCUSSION}

A numerical solution to the fully coupled problem of solving for the internal magnetic field in a three-dimensional rectangular prism with spatially varying temperature, applied magnetic field and magnetization has been derived and implemented. The model was applied to several relevant cases where the orientation of a magnetic material and an applied magnetic field is crucial combined with imposed temperature gradients. The magnetic material was assumed to be either homogeneous or a multilayered composite.

From the results presented in this paper it can be concluded that detailed knowledge of the demagnetizing field throughout the sample is important in many cases. This includes the situations when the temperature is not spatially constant or the sample is a composite material consisting of several materials each having a distinct Curie temperature. Imposing a temperature gradient across the sample makes the internal magnetic field become spacially asymmetric and especially when the demagnetization tensor field is rather large the internal field may be approximately linear as shown in Fig. 4(b). In this case the largest value of the internal field in the sample may be $50 \%$ greater than the smallest, which certainly invalidates any assumption of constant magnetization throughout the sample.

When applying a magnetic field along the direction of the grading of the material (in this case the $x$-direction) discontinuities on each internal boundary are observed. This is a direct consequence of the boundary conditions that apply generally for $\mathbf{H}$ and $\mathbf{B}$. This leaves two preferred directions to apply the magnetic field in (the $x$-direction and $y$-direction, respectively), in order to minimize the demagnetizing field. However, a large difference is observed in the behavior of the internal magnetic field between these two cases. When the external magnetic field is applied along the $x$-direction, discontinuities exist at every internal material boundary due to the boundary conditions for $\mathbf{H}$. In the other case, when the applied magnetic field is along the $y$-direction, no discontinuities are present. Furthermore, the magnitude of the internal magnetic field is generally seen to be larger in this case. It may therefore be concluded that great care should be taken when deciding along which direction the magnetic field should be applied with respect to both the demagnetizing field and a possible grading of the magnetic material.

In the case of applying the magnetic field in the $z$-direction to a constant temperature sample a difference between single and multiple material prisms is observed. In the former case the internal field is fairly constant. In the latter case the internal field becomes almost linear in the $x$-direction for a range of temperatures [see Fig. 5(c)]. However, when imposing a temperature gradient in the $x$-direction the virtually opposite is the case [compare Figs. 4(b) and 7(c)].

An average demagnetization factor was introduced and compared to the analytical expression calculated in Ref. 3 which is based on the assumptions that the magnetization is constant and completely aligned with the applied field. However, when the prism does not have a constant temperature or is made of a composite of different magnetocaloric materials, the demagnetization factor of Eq. (12) changes slightly.

Finally, it is concluded that the internal magnetic field is far from being constant under realistic circumstances and that it may be a poor approximation to assume so. As expected, when imposing a temperature gradient across the rectangular prism and assuming a composite material the internal field can become highly inhomogeneous, depending on the orientation of the applied field. Such inhomogeneities 
are important in any case where a good representation of the internal field is sought. It is noted that the results of this paper are valid for single prisms only. In many situations stacks or arrays of prisms will be relevant. A future paper on this using the model presented here is in preparation.

\section{ACKNOWLEDGMENTS}

The authors thank the support of the Programme Commission on Energy and Environment (EnMi) (Contract No. 2104-06-0032) which is part of the Danish Council for Strategic Research.

\section{APPENDIX A: CALCULATING THE D TENSOR FIELD}

The vector potential of a single magnetic dipole at $\mathbf{r}^{\prime}$ is

$$
\mathbf{A}_{i}(\mathbf{r})=\frac{\mu_{0}}{4 \pi} \frac{\mathbf{m}_{i} \times\left(\mathbf{r}-\mathbf{r}^{\prime}\right)}{\left|\mathbf{r}-\mathbf{r}^{\prime}\right|^{3}}
$$

The total vector potential of a magnetic body is obtained by integrating over the interior of the body (with $\mathbf{m}_{i}=\mathbf{M d} V$ ):

$$
\mathbf{A}(\mathbf{r})=\frac{\mu_{0}}{4 \pi} \int_{\Omega} \mathrm{d} \mathbf{r}^{\prime} \frac{\mathbf{M}\left(\mathbf{r}^{\prime}\right) \times\left(\mathbf{r}-\mathbf{r}^{\prime}\right)}{\left|\mathbf{r}-\mathbf{r}^{\prime}\right|^{3}}
$$

Note that this gives the vector potential both inside and outside of the prism.

The resulting $\mathbf{H}$-field is

$$
\begin{aligned}
\mathbf{H}(\mathbf{r}) & =\frac{1}{\mu_{0}} \mathbf{B}(\mathbf{r})-\mathbf{M}(\mathbf{r})=\frac{1}{\mu_{0}} \nabla \times \mathbf{A}-\mathbf{M}(\mathbf{r}) \\
& =-\frac{1}{4 \pi} \int_{\Omega} \mathrm{d} \mathbf{r}^{\prime}\left(\mathbf{M}\left(\mathbf{r}^{\prime}\right) \cdot \nabla\right) \frac{\mathbf{r}-\mathbf{r}^{\prime}}{\left|\mathbf{r}-\mathbf{r}^{\prime}\right|^{3}},
\end{aligned}
$$

which is the required demagnetizing field, $\mathbf{H}_{\mathrm{dem}}$.

The differentiations can be performed straightforwardly, giving rise to the following equation

$$
\mathbf{H}_{\mathrm{dem}}(\mathbf{r})=\frac{1}{4 \pi} \int_{\Omega} \mathrm{d} \mathbf{r}^{\prime} \mathrm{D}\left(\mathbf{r}-\mathbf{r}^{\prime}\right) \cdot \mathbf{M}\left(\mathbf{r}^{\prime}\right)
$$

with $\mathbb{D}$ being a symmetrical $3 \times 3$ tensor with elements

$$
\begin{aligned}
& D_{i i}(\mathbf{r})=-\frac{1}{|\mathbf{r}|^{3}}+\frac{3 x_{i}^{2}}{|\mathbf{r}|^{5}} \\
& D_{i j}(\mathbf{r})=\frac{3 x_{i} x_{j}}{|\mathbf{r}|^{5}}, \quad i \neq j
\end{aligned}
$$

Considering a rectangular prism with constant magnetization, $\mathbf{M}(\mathbf{r})=\mathbf{M}_{0}{ }^{4}$, the demagnetizing field becomes

$$
\begin{aligned}
\mathbf{H}_{\mathrm{dem}}(\mathbf{r}) & =\frac{1}{4 \pi} \int_{-a}^{a} \mathrm{~d} x^{\prime} \int_{-b}^{b} \mathrm{~d} y^{\prime} \int_{-c}^{c} \mathrm{~d} z^{\prime} \mathrm{D}\left(\mathbf{r}-\mathbf{r}^{\prime}\right) \cdot \mathbf{M}_{0} \\
& =-\mathbb{N}(\mathbf{r}) \cdot \mathbf{M}_{0},
\end{aligned}
$$

where the symmetric $3 \times 3$ demagnetization tensor $\mathbb{N}(\mathbf{r})$ has the components

$$
\begin{aligned}
N_{i i}(\mathbf{r})= & \frac{1}{4 \pi}\left[\arctan f_{i}(x, y, z)+\arctan f_{i}(-x, y, z)\right. \\
& +\arctan f_{i}(x,-y, z)+\arctan f_{i}(x, y,-z) \\
& +\arctan f_{i}(-x,-y, z)+\arctan f_{i}(x,-y,-z) \\
& \left.+\arctan f_{i}(-x, y,-z)+\arctan f_{i}(-x,-y,-z)\right]
\end{aligned}
$$

where

$$
\begin{aligned}
& f_{x}(x, y, z)=\frac{(b-y)(c-z)}{(a-x)\left[(a-x)^{2}+(b-y)^{2}+(c-z)^{2}\right]^{1 / 2}} \\
& f_{y}(x, y, z)=\frac{(a-x)(c-z)}{(b-y)\left[(a-x)^{2}+(b-y)^{2}+(c-z)^{2}\right]^{1 / 2}}
\end{aligned}
$$

$$
f_{z}(x, y, z)=\frac{(b-y)(a-x)}{(c-z)\left[(a-x)^{2}+(b-y)^{2}+(c-z)^{2}\right]^{1 / 2}} .
$$

The off-diagonal elements are

$$
N_{i j}(\mathbf{r})=-\frac{1}{4 \pi} \ln \left[\frac{F_{i j}(\mathbf{r}, a, b, c) F_{i j}(\mathbf{r},-a,-b, c) F_{i j}(\mathbf{r}, a,-b,-c) F_{i j}(\mathbf{r},-a, b,-c)}{F_{i j}(\mathbf{r}, a,-b, c) F_{i j}(\mathbf{r},-a, b, c) F_{i j}(\mathbf{r}, a, b,-c) F_{i j}(\mathbf{r},-a,-b,-c)}\right], \quad i \neq j
$$

where

$$
\begin{aligned}
& F_{x y}(\mathbf{r}, a, b, c)=(c-z)+\left[(a-x)^{2}+(b-y)^{2}+(c-z)^{2}\right]^{1 / 2} \\
& F_{y z}(\mathbf{r}, a, b, c)=(a-x)+\left[(a-x)^{2}+(b-y)^{2}+(c-z)^{2}\right]^{1 / 2}
\end{aligned}
$$

\section{APPENDIX B: NUMERICAL IMPLEMENTATION OF THE MODEL}

This appendix describes the implementation of a numerical model for solving the demagnetization problem as stated in Eqs. (3) and (7). First a simple scheme for optimized 


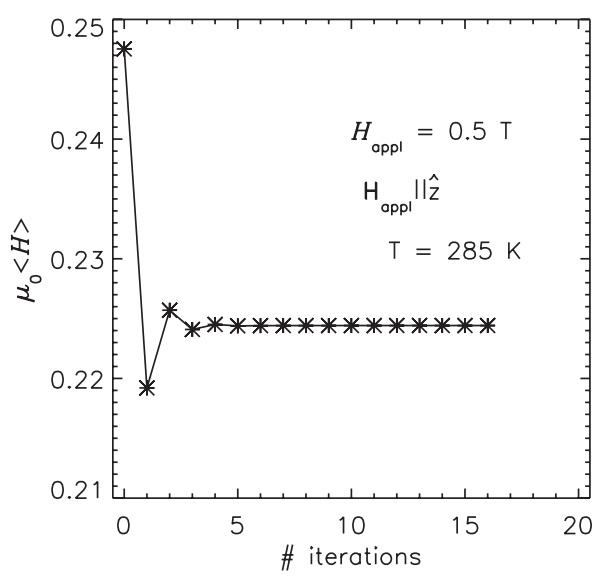

FIG. 9. The mean of the internal magnetic field as a function of number of iterations for the case when applying the magnetic field in the $z$-direction, setting the temperature to be constant at $285 \mathrm{~K}$ (below the Curie temperature) and $\mu_{0} H_{\text {appl }}=0.5 \mathrm{~T}$.

convergence conditions is presented. Second, the resolution of the model is discussed. Finally, symmetry conditions and parallelization are considered since the problem is of order $n^{2}$ with $n=n_{x} \times n_{y} \times n_{z}$.

\section{Convergence}

The criterium for convergence is defined as the maximum difference between the internal magnetic fields in two following iterations should be less than $10^{-8} \mathrm{~T}$. This is a criterium that is very similar to that of Ref. 8. An underrelaxation technique on the magnetization for obtaining convergence in situations with small applied fields and/or temperatures below the Curie temperature was applied in Ref. 8 . By thorough testing, we found that under-relaxing on the internal field was better for convergence. This may be expressed as

$$
\mathbf{H}_{n+1}=\mathbf{H}_{n}+\lambda_{n}\left[\mathbf{H}\left(M_{n}\right)-\mathbf{H}_{n}\right],
$$

where $n$ denotes the iteration step, $\mathbf{H}_{0}=\mathbf{H}_{\text {appl }}, M_{n}=M\left(H_{n}\right)$ using Eq. (8) and assuming $\mathbf{M}$ to be parallel to $\mathbf{H}$ in the previous step and $\mathbf{H}\left(M_{n}\right)$ is obtained through Eqs. (3) and (7). The under-relaxation parameter for the $n$th iteration is denoted $\lambda_{n}$, which attains a value in the interval $0<\lambda_{n} \leq 1$. Finally, it is noted that Eq. (B1) is used on every mesh point and the convergence is determined from the mesh point where two consecutive iterations yield $\max \left[\operatorname{abs}\left(\mathbf{H}_{n}-\mathbf{H}_{n+1}\right)\right]$ ensuring the slowest but most precise convergence. Figure 9 shows an example of the under-relaxation technique.

\section{Resolution}

A variation in resolution is shown in Fig. 10. The resolution of the prism is in all cases, except when comparing to the average demagnetization factor, $\left(n_{x}, n_{y}, n_{z}\right)=k(2 a, 2 b, 10$ $\times 2 c$ ) with $k$ being an arbitrary scaling constant. As can be seen from the figure a fairly low resolution is sufficient. This corresponds to $\left(n_{x}, n_{y}, n_{z}\right)=(20,20,10)$ for the case discussed in this work.
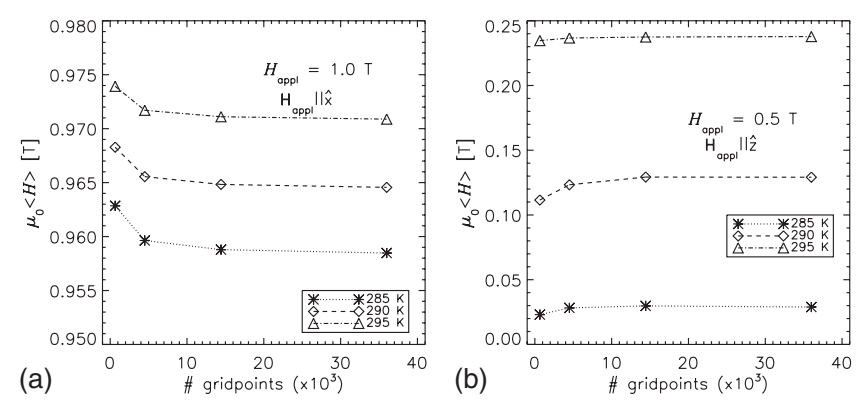

FIG. 10. The mean of the internal magnetic field as a function of the number of grid points for three different constant temperatures and an applied field of $1.0 \mathrm{~T}$ applied along the $x$-direction (a) and an applied field of $0.5 \mathrm{~T}$ applied along the $z$-direction (b)

\section{Symmetry and optimization}

The solution to the problem stated in Eqs. (3) and (7) both involves iteration of Eq. (3) and an $n^{2}$ problem from Eq. (7). Optimization in the form of exploitation of symmetry should be employed. The rectangular prism is symmetric around all three axes meaning that only one octant needs be considered when calculating the demagnetization tensor field, $\mathbb{N}$. Obviously, the applied field, temperature and magnetization cannot a priori be assumed to be symmetric since realistic scenarios include both temporally and spatially varying magnetic fields and temperatures.

However, the nature of $\mathbb{N}$ is purely geometric and is thus only a function of $\mathbf{r}-\mathbf{r}_{i, j, k}^{\prime}, a^{\prime}, b^{\prime}$, and $c^{\prime}$. Since the grid is defined to be homogeneous, the calculation of $\mathbb{N}$ only has to be performed once (at the beginning of the iteration process). Furthermore, $\mathbb{N}$ needs only to be evaluated in one octant and from this result can be mirrored to the remaining part of the coordinate system. Finally, during the calculations needed for one iteration, the value of $M$ is obtained from the previous iteration (or, in the case of the first iteration, from the initial guess), which means that the evaluations of the dot products between $\mathbb{N}$ and $\mathbf{M}$ needed in Eq. (7) are completely decoupled, which results in the possibility of maximized parallelization.

${ }^{1}$ P. M. Levy and D. P. Landau, J. Appl. Phys. 39, 1128 (1968).

${ }^{2}$ J. A. Osborn, Phys. Rev. 67, 351 (1945).

${ }^{3}$ A. Aharoni, J. Appl. Phys. 83, 3432 (1998).

${ }^{4}$ R. I. Joseph and E. Schloemann, J. Appl. Phys. 36, 1579 (1965).

${ }^{5}$ M. Beleggia and M. De Graef, J. Magn. Magn. Mater. 263, L1 (2003).

${ }^{6}$ S. Tandon, M. Beleggia, Y. Zhu, and M. De Graef, J. Magn. Magn. Mater. 271, 9 (2004).

${ }^{7}$ S. Tandon, M. Beleggia, Y. Zhu, and M. De Graef, J. Magn. Magn. Mater. 271, 27 (2004).

${ }^{8}$ J. A. Brug and W. P. Wolf, J. Appl. Phys. 57, 4685 (1985).

${ }^{9}$ O. Peksoy and A. Rowe, J. Magn. Magn. Mater. 288, 424 (2005).

${ }^{10}$ M. Lu, Z. Yang, and F.-L. Wei, Int. J. Infrared Millim. Waves 19, 1027 (1998).

${ }^{11}$ M. Schabes and A. Aharoni, IEEE Trans. Magn. 23, 3882 (1987).

${ }^{12}$ A. H. Morrish, The Physical Priciples of Magnetism (Wiley, New York, 1965).

${ }^{13}$ A. Rowe and A. Tura, Int. J. Refrig. 29, 1286 (2006).

${ }^{14}$ G. J. Liu, J. R. Sun, J. Z. Wang, T. Y. Zhao, and B. G. Shen, J. Phys.: Condens. Matter 19, 466215 (2007).

${ }^{15}$ D. R. Lide, CRC Handbook of Chemistry and Physics (CRC, Boca Raton, FL, 2004).

${ }^{16}$ A. M. Tishin and Y. I. Spichkin, The Magnetocaloric Effect and its Applications (Institute of Physics, London, 2003). 